3 Maestrelli $\mathbf{P}$, De Fina $O$, Bertin $T$, et al Integrin expression on neutrophils and mononuclear cells in blood and induced sputum in stable asthma. Allergy 1999;54:1303-8.

4 Alexis NE, Lay JC, Almond M, et al. Inhalation of low-dose endotoxin favors local $\mathrm{TH} 2$ response and primes airway phagocytes in vivo. J Allergy Clin Immunol 2004;114:1325-31

\section{Lymphangioleiomyomatosis- presence of receptor tyrosine kinases and the angiogenesis factor VEGF-A as potential therapeutic targets}

Lymphangioleiomyomatosis (LAM) is a rare systemic disorder in women occurring either sporadically (sporadic LAM) or in association with tuberous sclerosis (TS-LAM). It is caused by proliferating smooth muscle-like LAM cells, which lead to a progressive cystic destruction of the lungs and abdominal tumours (renal angiomyolipomas and/or axial lymph node lesions). LAM cells express receptors for oestrogen and progesterone and stain positive for HMB-45, an antibody against the melanoma-related antigen. ${ }^{1}$ LAM fulfils the criteria of a neoplastic disease with enhanced proliferation ${ }^{2}$ metastasising processes, increased migratory activity and invasiveness of LAM cells. ${ }^{4}$ Currently, an effective treatment interfering with these processes does not exist. Growth factors such as platelet-derived growth factor (PDGF) and epidermal growth factor (EGF) have been identified to enhance LAM and renal angiomyolipoma cell proliferation in vitro. ${ }^{25}$ Whether LAM cells express growth factorassociated receptor tyrosine kinases and the angiogenesis factor vascular endothelial growth factor-A (VEGF-A), which represent promising targets of small-molecule and antibody therapy in neoplastic diseases, is currently unknown.

We studied immunohistochemically the expression of the following proteins by LAM cells in 10 formalin-fixed and paraffin-embedded LAM specimens: epidermal growth factor receptor (EGFR; PharmDx Kit, Dako, Hamburg, Germany), platelet-derived growth factor receptor $\alpha$ (PDGFR- $\alpha$; rabbit polyclonal, Dianova, Hamburg, Germany), human epidermal growth factor receptor-2 (HER2; HercepTest, Dako), VEGF-A (clone VGl identifying the VEGF-A isoforms $\mathrm{VEGF}_{121}, \mathrm{VEGF}_{165}$ and $\mathrm{VEGF}_{189}$, DCS, Hamburg, Germany) and c-KIT (CD117; rabbit polyclonal, Dako). Staining procedures were carried out according to the manufacturer's instructions, and appropriate positive and negative controls were used. A semiquantitative scoring system of the immunohistochemical reactions for all receptor tyrosine kinases, the hormone receptors and VEGF-A was applied as follows: negative, no reaction or percentage of positive cells $<5 \%$; $1,5-25 \%$ positive cells; $2,26-$ $50 \%$ positive cells; $3,51-75 \%$ positive cells; 4 , $>75 \%$ positive cells; + , weak staining intensity; ++, moderate staining intensity; ++, strong staining intensity. Histological severity of lung destruction was assessed using the LAM histological score. ${ }^{6}$ The assessment of the LAM histological score and the immunohistochemical stainings was performed independently by two histopathologists (KE and MA). Only morphologically clear-cut, HMB-45 positive LAM lesions (nodules, cysts and diffuse LAM cell proliferations) were taken for analysis. All final decisions were made by consensus. Additionally, EGFR gene copy number per LAM cell nucleus was investigated by one histopathologist (SL) using fluorescence in situ hybridisation (FISH; LSI EGFR SpectrumOrange/ CEP 7 SpectrumGreen probe, Vysis, Abbott Laboratories, Wiesbaden, Germany). The study

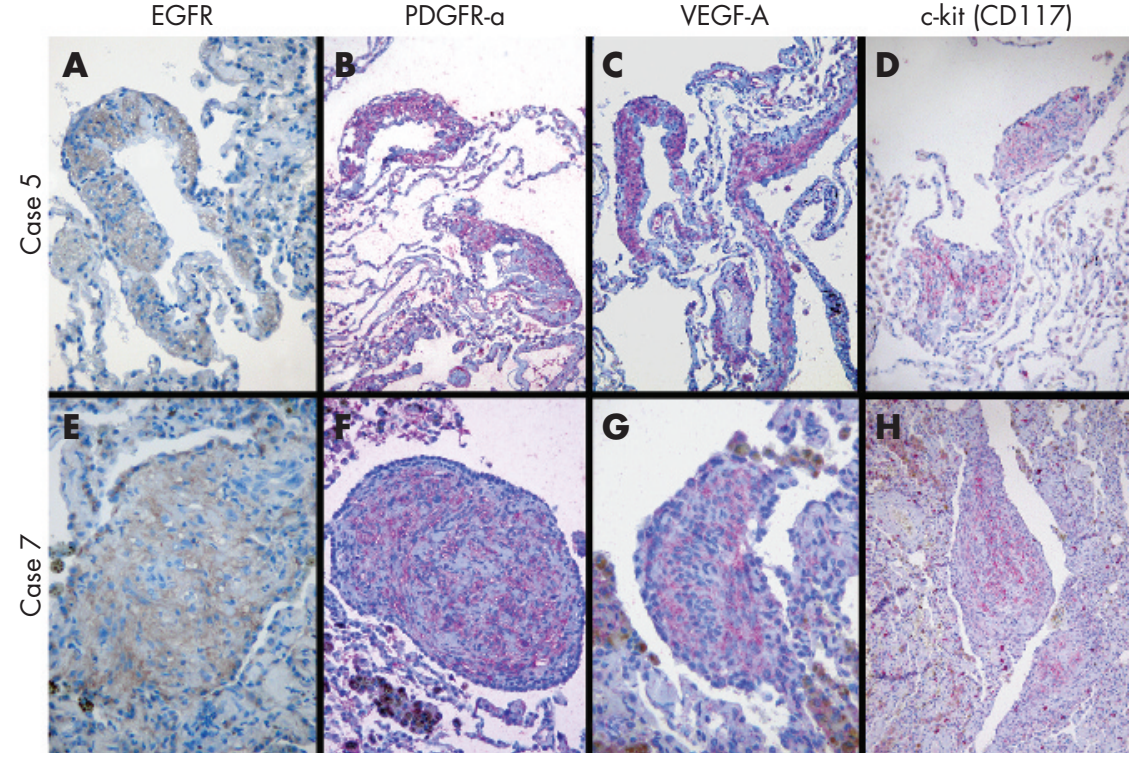

Figure 1 Expression of epidermal growth factor receptor (EGFR), platelet-derived growth factor receptor $\alpha$ (PDGFR- $\alpha$ ), vascular endothelial growth factor-A (VEGF-A) and c-KIT (CD117) in lung lymphangioleiomyomatosis (LAM) lesions. The panel shows two pulmonary LAM specimens (case 5, $A-D ;$ case $7, E-H)$. Case 5 (A-D) represents predominant cystic and diffuse proliferating LAM lesions, whereas case $7(E-H)$ represents predominant nodular growth pattern. The cases show a variable expression of EGFR, PDGFR- $\alpha$, VEGF-A and c-KIT (CD117).

was approved by the local ethics committee and written informed consent was obtained from al participants or their close relatives.

In all specimens, LAM lesions were consistently positive for PDGFR- $\alpha$ and VEGF-A. EGFRpositive LAM cells were observed in seven specimens. No amplification or higher polysomy of the EGFR gene was detected. In addition to cKIT-positive mast cells, which were sporadically present in LAM lesions and the surrounding lung tissue, LAM cells themselves were found to be positive for c-KIT in six of the specimens. HER2 was negative in all specimens (fig 1). For details, see supplementary table available online at http:/ www.thorax.bmjjournals.com/supplemental.

We demonstrated that PDGFR- $\alpha$, EGFR, c-KIT and VEGF-A as targets of currently available compounds are expressed by LAM cells. These findings imply further research in the field of small-molecule and antibody therapy in LAM.

\section{Acknowledgements}

We thank Professor H Morr, Dr J Linke, Dr J Galle, Dr C Smaczny and Dr M Choschzick for providing us with the tissue samples. We also thank Ralf Lieberz and his team for the excellent technical assistance.

Henrik Watz*

Pulmonary Research Institute, Hospital Grosshansdorf Center for Pneumology and Thoracic Surgery, Grosshansdorf, Germany

Knut Engels*

Department of Pathology, University Clinic Frankfurt, Frankfurt, Germany

Siegfried Loeschke

Department of Pathology, Resed Borstel, Germany

Michael Amthor

Department of Pathology, Hospital Rotenburg Rotenburg, Germany

Detlef Kirsten, Helgo Magnussen

Hospital Grosshansdorf, Center for Pneumology and Thoracic Surgery, Grosshansdorf, Germany
Helgo Magnussen

Pulmonary Research Institute, Hospital Grosshansdorf Center for Pneumology and Thoracic Surgery,

Grosshansdorf, Germany

Correspondence to: Dr H Watz, Pulmonary Research Institute, Hospital Grosshansdorf, Center for Pneumology and Thoracic Surgery, Woehrendamm 80, D-22927 Grosshansdorf, Germany h.watz@pulmoresearch.de

doi: 10.1136/thx.2006.071811

*These authors contributed equally to this work.

Competing interests: None.

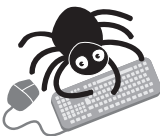

A supplementary table is available online at http:// www.thorax.bmi.com/ supplemental

\section{References}

Johnson SR. Lymphangioleiomyomatosis. Eur Respir J 2006;27:1056-65.

2 Goncharova EA, Goncharov DA, Spaits M, et al. Abnormal growth of smooth muscle-like cells in lymphangioleiomyomatosis: role for tumor suppressor TSC2. Am J Respir Cell Mol Biol 2006;34:561-72.

3 Crooks DM, Pacheco-Rodriguez G, Decastro RM, et al. Molecular and genetic analysis of disseminated neoplastic cells in lymphangioleiomyomatosis. Proc Natl Acad Sci USA 2004;101:17462-7.

4 Goncharova EA, Goncharov DA, Lim PN, et al. Modulation of cell migration and invasiveness by tumor suppressor TSC2 in lymphangioleiomyomatosis. Am J Respir Cell Mol Biol 2006;34:473-80.

5 Lesma E, Grande V, Carelli S, et al. Isolation and growth of smooth muscle-like cells derived from tuberous sclerosis complex- 2 human renal angiomyolipoma: epidermal growth factor is the required growth factor. Am J Pathol 2005; 167:1093-103

6 Matsui K, Beasley MB, Nelson WK, et al. Prognostic significance of pulmonary ymphangioleiomyomatosis histologic score. Am J Surg Pathol 2001;25:479-84. 